\title{
Managing Dynamics in Corporate Networks
}

\author{
Mike Danilovic ${ }^{1,2}$, Mats Winroth ${ }^{3}$ \\ ${ }^{1}$ Centre of Innovation, Entrepreneurship and Learning Research, School of Business and Engineering, \\ Halmstad University, Halmstad, Sweden \\ ${ }^{2}$ Shanghai Dianji University, Shanghai, China \\ ${ }^{3}$ Division of Operations Management, Department of Technology Management and Economics, Chalmers \\ University of Technology, Gothenburg, Sweden \\ Email: mike.danilovic@hh.se, mats.winroth@chalmers.se
}

Received 9 November 2013; revised 17 December 2013; accepted 26 December 2013

Copyright (c) 2014 Mike Danilovic, Mats Winroth. This is an open access article distributed under the Creative Commons Attribution License, which permits unrestricted use, distribution, and reproduction in any medium, provided the original work is properly cited. In accordance of the Creative Commons Attribution License all Copyrights (C) 2014 are reserved for SCIRP and the owner of the intellectual property Mike Danilovic, Mats Winroth. All Copyright (c) 2014 are guarded by law and by SCIRP as a guardian.

\begin{abstract}
A crucial issue in collaborating in manufacturing corporate networks between different companies is to identify to what extent different strategic and operational decisions need to be coordinated between the involved companies. In this paper, we elaborate on the issue of synchronization and coordination of information flow based on interconnectivities between companies in order to coordinate a corporate network by the means of DSM, Dependence Structure Matrix. The results show that DSM can be used to identify interconnectivities, dependencies on information flow among actors in a network and to identify which information needs to be shared between companies in the network.
\end{abstract}

\section{Keywords}

\section{Corporate Network; Supplier Collaboration; Design Structure Matrix}

\section{Introduction}

The competitive situation for many small- and medium-sized companies, often acting as suppliers to larger companies, has become more intense in terms of reduced lead times in product development and manufacturing, cost reductions, and increased demands for higher product quality. The stiffer competition has also forced systems integrators to develop new strategies to meet new demands. Management issues, such as lean production, system strategies, and outsourcing, have been introduced and over time become dominant ideas of a changed direction among many corporations [1,2]. If attributes, such as a high level of customization, short lead times in 
product development and quick responses to late changes of specifications are important, long distances and perpetual changes of suppliers may cause disturbance.

However, from the literature we can see that relations between systems integrating companies and its suppliers are handled in different ways. Brandes et al. [3] discussed the reduction of the supplier base in terms of the number of suppliers. The selected suppliers are subject to increased demands to participate in the process of product development with the systems integrating corporation and their other suppliers on different supplier levels. The expectation that suppliers participate in the process of innovation and delivery of complete products or subsystems was further discussed [4-6]. These new demands on suppliers from systems integrators to collaborate have more intensively forced small- and medium-sized corporations to find new organizational settings. How can a small company adapt to these new demands with its scarce resources and limited knowledge base? One solution is that several companies work together, acting as one unit outwardly. However there may be a drawback in these close collaborations. Gulati et al. [7] described the so-called lock-in and lock-out effects. These constraints are both consequences of close collaboration with one actor. The lock-in effect is the result of limited resources and implies that a company only has time and resources to form and satisfy the expectations of a limited number of alliances. The lock-out effect is the result of alliances that request total loyalty of its members on the contrary. The consequence of these two constraints could be that companies have to reject collaboration with new partners.

\section{Research Approach and Research Methodology}

Our approach in this research is to apply an analysis model originally based on research of relations between manufacturing strategy and design of manufacturing system within a single company [8,9]. This model has been extended in the analysis of a dyadic relation between two companies [10,11]. In this research we investigated how this analytical model can be applied to a network setting, in which one systems integrator have close collaboration with three suppliers. This research, presented in this paper, is based on a series of interviews with people at all four companies involved in a collaborative network. We have also studied how this manufacturing network works in order to produce and deliver the complete systems to customers. We have performed four interviews that have been recorded, typed and double checked with the interviewees. Mapping of corporate strategy and their manufacturing design presented in this paper have been performed on the basis of collected information in interviews and is thus our interpretation of the circumstances at hand within each company and between companies.

\section{Case Study}

The systems integrating (SI) company in this case study, Kalmar Industries, is part of a large multinational industrial group, with its head office outside Sweden. The SI has approximately 350 employees. The total turnover at the SI in 2002 was approximately EUR 760 million.

A new great business deals in development, manufacturing, and delivering a number of large-scale lifters was made with a foreign defense authority. This new large business deal was supposed to accommodate 200 people for a period of several years. In addition, this new customer demanded that the supplier of these new heavy lifters, the SI, should take tighter control over the manufacturing facilities and processes of development, manufacturing, and delivery of completed products.

The ability to create a highly flexible organization demands that major suppliers actively take part in the development, manufacture, and delivery of final products to customers. One solution is to increase the number of tasks that the key suppliers could perform. Since one of the most important competitive factors is flexibility in accepting very late changes of product specifications, the delivery lead times from the suppliers would be too long. The solution to this situation was a reallocation of suppliers' manufacturing units into a mutual Product Supply Centre (PSC).

Supplier A supplies hydraulic hoses, supplier B delivers the tires for the container handlers, and supplier C provides the hydraulic cylinders to the trucks. The reasons for integrating these suppliers vary, but one common driving factor was that geographic closeness would improve communication and reduce lead times.

\section{Approaches to Manufacturing Strategies and Production Systems Design}

The issue of how the manufacturing strategies and the design of the production system influence each other was 
investigated by e.g. Miltenburg [8]. The original analysis framework was built up by three main parts: a table showing the competitive situation of the company (or business area), a diagram classifying the production system into one of seven production systems and showing the support for different competitive factors, and a list of six decision categories that together show the manufacturing strategy of the company. They were graded according to a four-degree scale, ranging from internally neutral to externally supportive (or "infant" to "worldclass"), i.e. how they support the overall business strategy of the company [12]. Säfsten and Winroth [9] elaborated further on the analysis model and came to the conclusion that the framework worked in general quite well for single organizations. Miltenburg [13] also presented an alternative solution of the framework dealing with the plant-within-a-plant problem. This approach could be relevant also for collaborative settings, since the issue is to prevent information leakage between parts of the plant working towards competing customers.

Companies are, however, open systems and there are always input and output relationships with the environment. In network settings several companies have to collaborate and each of these companies needs to have a set of manufacturing strategies comprising different design production systems.

\section{Empirical Investigation of a Collaborative Network}

A prerequisite for achieving competitive advantages in network settings is that companies must be supportive to each other regarding supply capability. The individual corporate manufacturing strategy and production systems should be coordinated in order to together support the overall aims of the network. At the same time, the actors of a collaborative network setting are often involved in other networks or supplying a specific customer. This means that they cannot focus the design of the production system and support functions totally on just one situation. They must keep the flexibility thus making trade-offs in order to be able to continue to produce a variety of products or parts. A wider presentation of different issues around network collaboration, necessary organizational actions to take, and factors important to the network if it will succeed was made by Winroth and Danilovic [10]. In our analysis we started with the extended Miltenburg model. In the Miltenburg model manufacturing design is influenced by the manufacturing output and competitive situation within each company of the network. However, in the network settings those aspects have to be synchronized across companies of the network. The network based Miltenburg analysis should therefore precede several steps.

1) First each company performs an analysis of their own manufacturing strategy and production system according to Säfsten and Winroth [9]. The conditions between the SI and each network partner are then synchronized in a number of steps.

2) The SI correlates its competitive priorities with its production system and detects suitable changes.

3) The SI performs an analysis of its strategic decision categories and makes suitable changes, either to the production system design or the decision categories.

4) The SI's strategic decision categories give input to the decision categories of Suppliers A, B, and C.

5) Each supplier correlates its competitive priorities with its production system and detects suitable changes.

6) Each supplier performs an analysis of its strategic decision categories and makes suitable changes, either to the production system design or the decision categories.

7) The suppliers coordinate their decision categories and take necessary actions.

In our approach to explore the linkages between manufacturing strategies and production system in a collaborative network setting we identified three major processes, loops of information processing, developing a joint manufacturing strategy for the collaborative network and design of production systems within each company enabling manufacturing and delivery of complete products to final customer, as shown in Figure 1.

The first loop is a process mainly taking place at the systems integrator level (SI), involving steps 1 and 2 . As the leading actor in creating the network setting, the SI has to analyze the market situation and the customer requirements. In this process the present manufacturing systems influence the analysis of decisions areas, which is fed back to the design of the production system. In this case the new customer placed increased demands on the SI to deliver complete products, while the main shareholders placed financial restrictions.

The second loop is a process of synchronization between SI and preferred suppliers. In this synchronization, SI and its suppliers are expected to communicate market situation and customer demands and to negotiate how suppliers should organize their production systems according to what SI was capable of doing on its own and what part of the supplier organization should be relocated to SI area.

The third loop is a process of adaptation on the supplier level within each preferred supplier and also mutual 


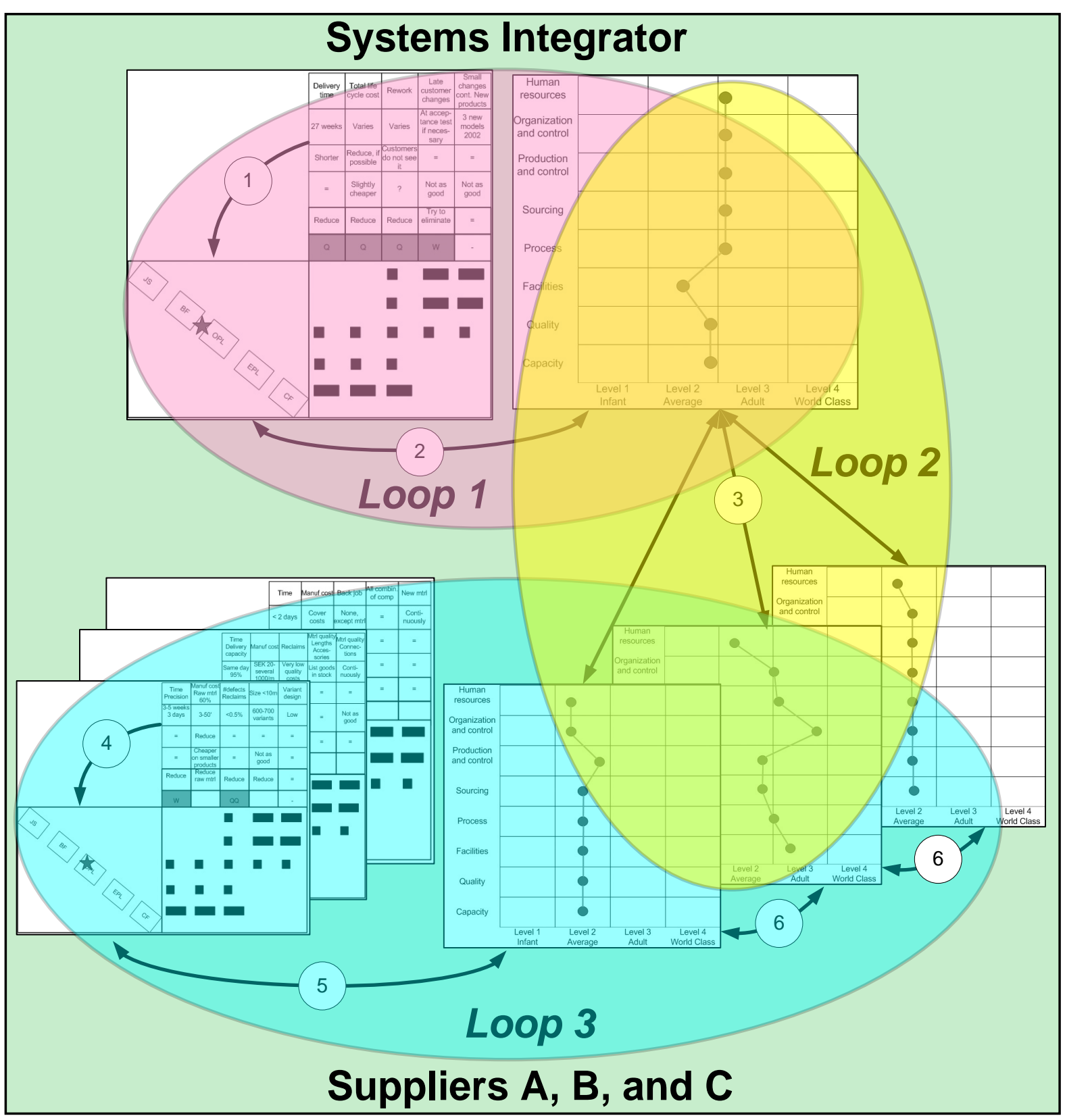

Figure 1. Coordination loops between systems integrator and suppliers.

adjustments between suppliers. They have to decide how to respond to the demands, what part to relocate, and how to develop new organizational routines to handle the daily life activities not only in their own corporation but also within the entire network.

The methodology that is used to represent and analyze dependencies and relations between items is known as Design Structure Matrix or Dependence Structure Matrix (DSM) and was introduced by Steward [14]. As a matrix structure is used to represent the problem structure [15], two different kinds of matrices are used. One is square matrix and the other a rectangular matrix. The square matrix is used to represent one domain problem structure while the rectangular matrix is used to represent interactions between items in-between two different sets of items in two different domains. While the first is named as DSM the other one is named as Domain Mapping Matrix (DMM). In complex situations, combinations of DSM and DMM can enrich the analysis and help to analyze the dynamics of complex systems. DSM represents and visualizes interdependencies and relations be- 
tween items such as tasks and activities, components and subsystems, and among people and teams [16,17].

A DSM-based analysis shows how the design of tasks, sequencing of activities can be organized for the effective problem solving in team-based work and the communication required within and between teams [15,18-22].

DSM-STEP 1: Step 1 in our analysis is to outline all aspects of the Miltenburg model, as shown below, and put all those three aspects of competitive analysis, decision criteria, and production layout for all companies of the network in a matrix. In this matrix all rows and columns contain the same information.

DSM-STEP 2: The matrix containing aspects of the Miltenburg model from all four involved companies is a matrix of 162 rows and columns. Each row meets 162 columns. Each of those meeting points is called point of interaction (POI). Each POI contains information about interconnectivities/interdependencies between elements in the row and column. On the overall level there are 26244 POIs in our network matrix.

During interviews and workshops with people from all four companies of the network all those POIs were investigated if they hold any interconnectivity. In each POI, numbers and colors are used to show identified interconnectivity. No 1, marked with yellow color, shows low level of interconnectivity. No 2, with magenta color, shows medium high interconnectivity, and No 3, with red color, show high level of interconnectivity. Colors are used to enable visualization of patterns of interconnectivities.

Figure 2 shows the entire network matrix, filled with identified interconnectivities. On the network level this large matrix can be seen and handled as a large DSM matrix. In the figure we have also marked a number of sub-areas, in total 16 sub-areas. Each sub-area of the entire network matrix is either a DSM or DMM. Along the diagonal, four DSMs are outlined containing interconnectivities between aspects of the Miltenburg model but within each of the four companies of the network. The other sub-areas are 12 DMMs containing interconnectivities between companies of the network as shown in the matrix. The large network matrix can be seen as a hierarchical system of a number of sub-systems that can be extracted from the large matrix.

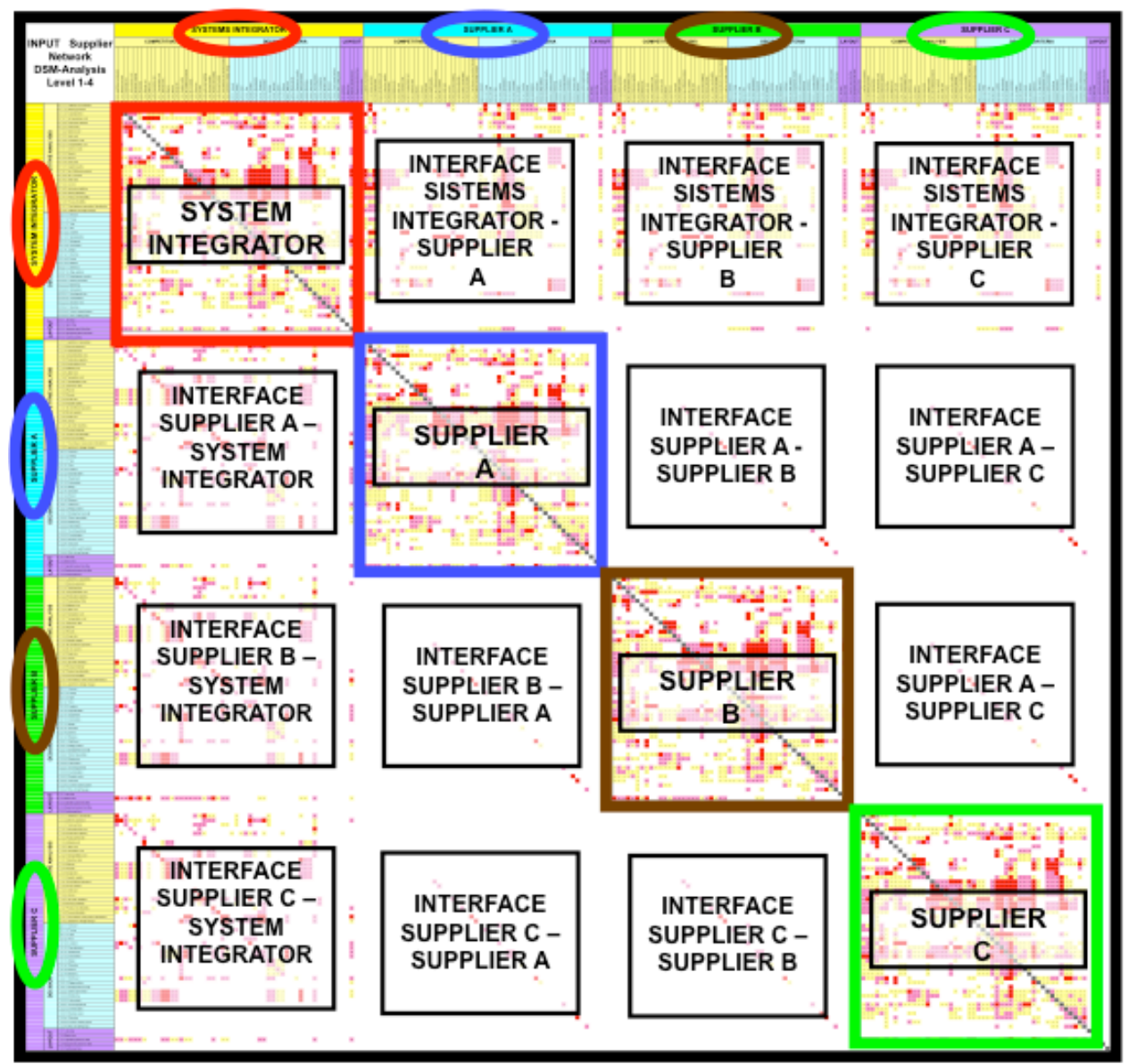

Figure 2. Overall picture of the network. 
The analysis of such a matrix is performed in specially designed software packages for matrix-based analysis. In our research we have worked with Complex Problem Solver that we have developed in order to support our research. Depending on whether it is a DSM sequencing, DSM clustering, or DMM clustering analysis that is expected, the software uses different algorithms. In this situation, focusing on the entire network, a DSM clustering approach was chosen in the first place.

In the clustered matrix, rows and columns are moved in such way that as many as possible of POI holding numbers are moved towards the diagonal. Along the diagonal we identify five clusters, high density of interconnectivities, between elements of the matrix. Clusters are numbered from 1 to 5 starting at the top left position and going to the lower right position. This numbering will be used in the cluster analysis below.

DSM-STEP 3: Generally it can be noted from this first clustering that a more automated and continuous flow system performs better, but it also increases the demands on the support functions. Efforts in managing quality give result.

After the major clusters have been identified, each cluster has been clustered in order find sub-clusters within the major clusters. This analysis has been performed on 16 DSM and DMM clusters. However, in this appear we only present the outcome of the cluster 3 , which we consider the most interesting one because it contains interconnectivities between all four companies of the corporate network, and we will also some concluding comments on the other clusters.

Cluster one emphasizes that quality and lead-time are extremely important and highly affect the production cost. In fact, cluster one does not include that much of network aspects but is more focused on internal issues within a company.

Cluster two deals also mostly with internal issues but also some aspects between supplier and SI. Location, automation level, and different flexibility aspects are strongly interlinked. We can also see that information regarding time aspects, layout, and no of variants etc are strongly influencing each other.

Cluster three, Figure 3, is more interesting especially in the lower right hand corner, where we can see the importance of inter-organizational efficiency and performance. All links need to perform well if the market requirements are to be met. The result shows that information sharing needs to be performed not only between SI and suppliers but also between the suppliers.

Cluster four focuses on internal aspects at the supplier A, capabilities and output such as productivity and cost, the choice of technology, location, and organization of production, quality management with the purpose of meeting the market demands, and the linkages to suppliers' quality are strong!

Cluster five focuses on suppliers A - C. Suppliers need to be involved in new product introductions, choices of location and vertical integration, location close to upstream suppliers or close to downstream customers, shows the links between decision criteria internally at SI, and a certain linkage between process flexibility and the quality of the inlet material.

\section{Conclusions}

In our approach we have used a matrix-based analysis of the interconnectivities between elements of the Miltenburg model within and between companies of a collaborative network. In order to function efficiently, it is crucial to understand what kind of information that different actors need and how each other's competitive advantage is influenced by strategic decisions, actions and interconnectivities. The combination of DSM and DMM approaches used in this research explores and enables synchronization, within and between corporations of the corporate network, regarding aspects of their intra-corporate and inter-corporate: Competitive situation (strategic), Decision criteria (tactical), and Layout of production system (operational).

This participative approach that we have chosen, involving people from all companies of the network involves several functions in the analysis and strategizing processes and reveals assumptions regarding market and competitive situation for involved corporations, explores the need for adaptation to customer needs, and the need for inter-corporate adaptation. Finally, this approach reduces uncertainty in decision-making, organizational and process design and enables development of self-organizing network settings. In our analysis of linkages between manufacturing strategies and production system in a collaborative network setting, we could identify three major processes: loops of information processing, developing a joint manufacturing strategy for the collaborative network, design of production systems within each company enabling manufacturing and delivery of complete products to final customer. 


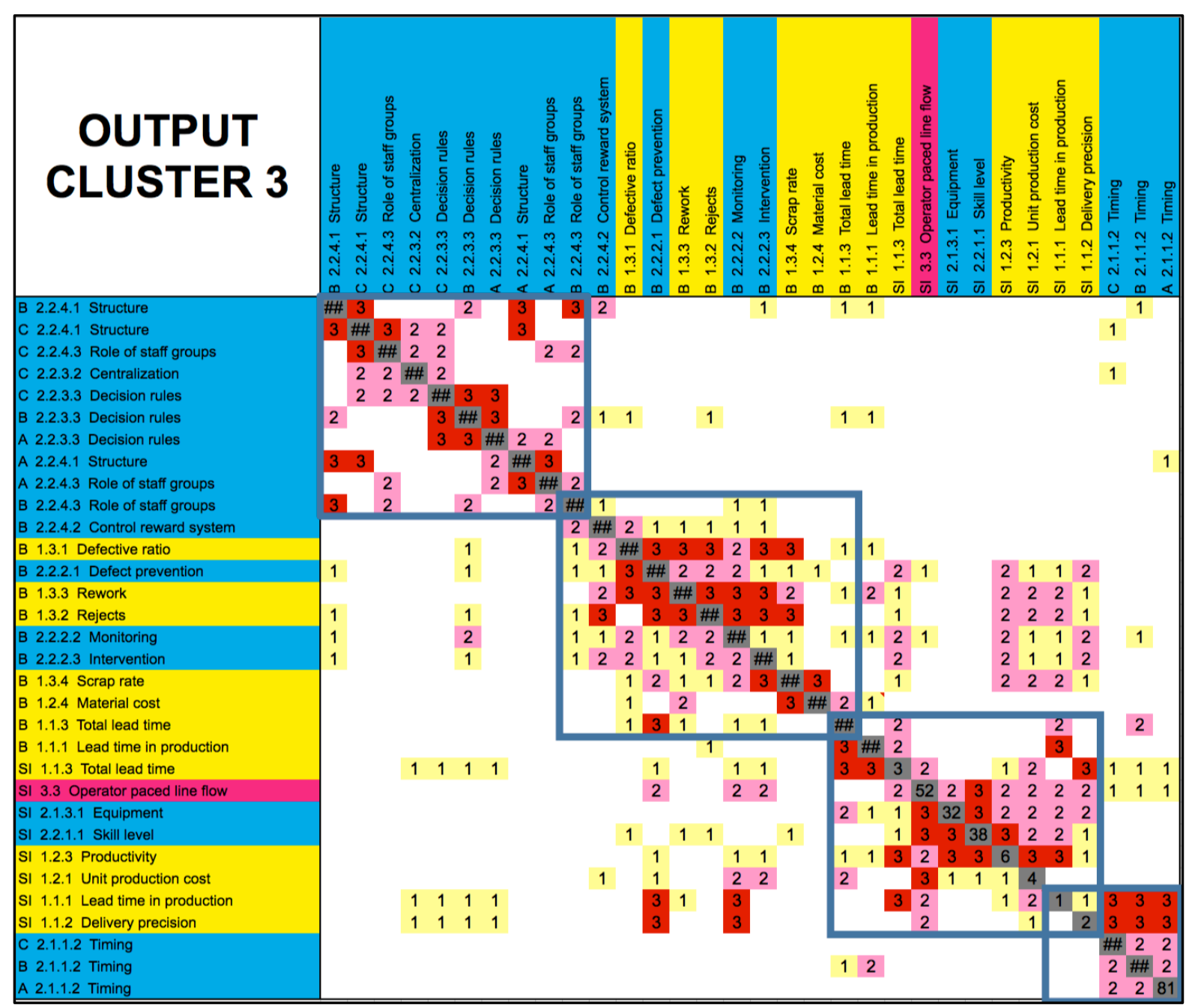

Figure 3. Output from cluster analysis 3.

The first loop is a process mainly taking place at SI level. Traditionally SI is performing strategic decisions based on their own understanding of business situation and market conditions, designing the manufacturing system and then ordering subsystems from independent suppliers. These chosen suppliers have limited access and understanding of how and why SI is acting as they are. The business situation of financial restrictions forced SI to look for another organizational solution to be capable of delivering complete products without increasing its own organization. The solution was to select few suppliers as their preferred suppliers. In addition to this, these preferred suppliers were asked to be collocated with SI. The reason was that SI had a vision of close collaboration with suppliers on the close range to enable intense communication and development of close relations between people in the supply chain. The chosen suppliers had to develop a joint manufacturing strategy together with the SI and other suppliers. In this new situation, SI and its suppliers had to synchronize their normally independent analysis into joint and mutually understood analysis and decisions that how to organise and operate business in order to suit each other's competitive situation, manufacturing design etc.

The second loop is a process of synchronization between SI and preferred suppliers. This turned in our analysis to be a crucial process. In this synchronization, SI and its suppliers were able to communicate market situation and customer demands. Also, they were forced to negotiate how suppliers should organize their production systems according to what SI was capable of doing on its own and what part of the supplier organization should be relocated to SI area. In the beginning of this process, not all suppliers did know much of each other. This synchronization process not only linked SI with suppliers but also linked suppliers with each other in a mutual process of negotiating, learning, and developing trust in each other. All participants came to understand that the 
final success was not only a question of SI as the corporation fronting the customer, but also rather a question for everybody. This synchronization process is what Westley [23] calls the strategic dialogue.

This appeared to be important managerial aspects of creating prerequisites for handling the unpredictable, the unknown, and the insecure that always characterize development and manufacturing of complex products. Management at SI and its suppliers in the corporate network developed an opportunity to analyze the present situation according to the mission, goals, and restrictions not only within the SI but also within each supplier and between them all as a system. When these aspects were discussed, management could mutually shape an appropriate strategy for conducting tasks out of common understanding and acceptance of conditions for this new situation. This process is labeled by Westley [23] as a strategic conversation and micro dynamics of inclusion. In addition, they can together design appropriate structures and processes. The subjectivity in the actor approach becomes a line of activities [24,25].

\section{Managerial Implications}

When network based solutions are chosen, some important questions need to be handled. To confront these issues, a systems approach is suggested starting with three major questions [26]:

1) The first question is who designs the system or the process.

2) The second question concerns the system evolution and the processes of change.

3) The third question considers the role of management.

The first question points out who is designing the system and the process for organizational and project design. We suggest that a participative approach should be used in which people from all participating companies in the network have to be involved. This is a strategic dialogue; they themselves are able to outline the design of this information exchange process.

The second question is on the nature of the system and its management. According to the systems approach of Buckley [26], we can imply that the manufacturing processes on the network level are equifinal, i.e. the desired results may be reached by different trajectories with varying costs and time requirements. However, the process of organizing is multi-final. The planning or the managerial process does not control the progress of the product development process. Different trajectories are likely to produce different outcomes. Thus there are no ways to specify in advance a procedure that will guarantee the desired outcomes. A participative approach enables those involved to design the organization of each project and the means of communication.

The third question focuses on management and its role in this participative process. The structure and quality of the communication system are key aspects [23]. Management in a company should use strategic conversation, discrete communication about strategic generalities of the network according to overall goals and milestones and day-to-day activities between SI and suppliers and between suppliers, as a means of supporting and maintaining the dynamics between companies in the collaborative network setting. This process of inclusion creates mutual understandings and feelings of belonging, brings clarity in areas of authority and responsibility, and helps in providing information about issues of boundary management. Thus they are now becoming companions and partners. The strategic conversation is a vital mean of supporting the dynamic behavior within each company and between companies. In this strategic conversation, people can perceive the picture of the world, within and outside their company or the network. The outcome should be a shared understanding of what needs to be done and why. For managers this process will create more time to shift focus from managing daily routines and giving orders to thinking globally and strategically.

\section{Acknowledgements}

The authors would like to thank the participating company, Kalmar Industries, for their kindness to participate. This study is also supported by Chalmers Area of Advance on Sustainable Production and its Sustainable Production Initiative.

\section{References}

[1] Womack, J.P., Jones, D.T. and Ross, D. (1990) The machine that changed the world. MIT Press, Cambridge.

[2] Ford, D., Cotton, B., Farmer, D., Gross, A.C. and Wilkinsson, I. (1993) Make-or-buy decisions and their implications. 
Industrial Marketing Management, 22, 207-214. http://dx.doi.org/10.1016/0019-8501(93)90007-T

[3] Brandes, H., Lilliecreutz, J. and Brege, S. (1997) Outsourcing-Success or failure? European Journal of Purchasing and Supply Management, 3, 63-75. http://dx.doi.org/10.1016/S0969-7012(97)00001-4

[4] von Hippel, E. (1988) The sources of innovation. Oxford University Press, Oxford.

[5] Clark, K.B. and Fujimoto, T. (1991) Product development performance: Strategy, organization, and management in the world auto industry. HBS Press, Boston.

[6] Quinn, J.B. (2000) Outsourcing innovation: The new wheel engine of growth. Sloan Management Review, 13-28.

[7] Gulati, R., Nitin, N. and Akbar, Z. (2000) Strategic networks. Strategic Management Journal, 21, 203-215. http://dx.doi.org/10.1002/(SICI)1097-0266(200003)21:3<203::AID-SMJ102>3.0.CO;2-K

[8] Miltenburg, J. (1995) How to formulate and implement a winning plan. Productivity Press, Portland.

[9] Säfsten, K. and Winroth, M. (2002) Analysis of the congruence between manufacturing strategy and production system in SMME. Computers in Industry, 49, 91-106. http://dx.doi.org/10.1016/S0166-3615(02)00061-1

[10] Winroth, M. and Danilovic, M. (2002) Manufacturing strategies_Congruence of manufacturing processes within a supply chain. Proceedings of the 13th Annual Conference of the Production and Operations Management Society, San Francisco, 5-8 April 2002.

[11] Danilovic, M. and Winroth, M. (2006) Corporate manufacturing network: From hierarchy to self-organising system. International Journal of Integrated Supply Management, 2, 106-131. http://dx.doi.org/10.1504/IJISM.2006.008341

[12] Hayes, R. and Wheelwright, S. (1984) Restoring our competitive edge: Competing through manufacturing. John Wiley \& Sons, New York.

[13] Miltenburg, J. (2008) Setting manufacturing strategy for a factory-within-a-factory. International Journal of Production Economics, 113, 307-323. http://dx.doi.org/10.1016/j.ijpe.2007.09.001

[14] Steward, D. (1981) The design structure system: A method for managing the design of complex systems. IEEE Transactions on Engineering Management, EM-28, 71-74. http://dx.doi.org/10.1109/TEM.1981.6448589

[15] Eppinger, S., Whitney, D., Smith, R. and Gerbala, D. (1994) A model-based method for organizing tasks in product development. Journal of Research in Engineering Design, 6, 1-13. http://dx.doi.org/10.1007/BF01588087

[16] Danilovic, M. and Sandkull, B. (2005) The use of dependence structure matrix and domain mapping matrix in managing uncertainty in multiple project situations. International Journal of Project Management, 23, 193-203. http://dx.doi.org/10.1016/j.ijproman.2004.11.001

[17] Danilovic, M. and Browning, T. (2007) Managing complex product development projects with design structure matrices and domain mapping matrices. International Journal of Project Management, 25, 300-314. http://dx.doi.org/10.1016/j.ijproman.2006.11.003

[18] Smith, R., Eppinger, S. and Gopal, A. (1992) Testing an engineering design iteration model in an experimental setting. Design Theory and Methodology, 42, 269-276.

[19] Smith, R. and Eppinger, S. (1997) Identifying controlling features of engineering design iteration. Management Science, 43, 276-293. http://dx.doi.org/10.1287/mnsc.43.3.276

[20] Morelli, M., Eppinger, S. and Gulati, R. (1995) Predicting technical communication in product development organizations. IEEE Transactions on Engineering Management, 42, 215-222. http://dx.doi.org/10.1109/17.403739

[21] Browning, T. (1997) Exploring integrative mechanisms with a view toward design for integration, advances in concurrent engineering-CE97. Fourth ISPE International Conference on Concurrent Engineering: Research and Applications, Rochester, 20-22 August 1997, 83-90.

[22] Browning, T. (2001) Applying the design structure matrix to system decomposition and integration problems: A review and new directions. IEEE Transactions on Engineering Management, 48, 292-306. http://dx.doi.org/10.1109/17.946528

[23] Westley, F.R. (1990) Middle managers and strategy: Microdynamics of inclusion. Strategic Management Journal, 11, 337-351. http://dx.doi.org/10.1002/smj.4250110502

[24] Garnsey, E. (1992) Critical systems thinking as an integrative perspective in social theory. University of Cambridge, Cambridge.

[25] Garnsey, E. (1993) Cumulative knowledge, determinism, and critical systems thinking. 11th EGOs Colloquium, Working Group on Epistemology of Managerial and Organizational Knowledge, Paris, 6-8 July 1993.

[26] Buckley, W. (1967) Sociology and modern systems theory. Prentice-Hall Inc., Englewood Cliffs. 